\title{
Video Article \\ Transplantation of Zebrafish Pediatric Brain Tumors into Immune-competent Hosts for Long-term Study of Tumor Cell Behavior and Drug Response
}

\author{
Mattie J. Casey ${ }^{* 1}$, Katarzyna Modzelewska* ${ }^{1}$, Daniela Anderson ${ }^{1}$, James Goodman ${ }^{1}$, Elena F. Boer ${ }^{1}$, Laura Jimenez ${ }^{1}$, Douglas Grossman ${ }^{1,2}$, \\ Rodney A. Stewart ${ }^{1}$ \\ ${ }^{1}$ Department of Oncological Sciences and Huntsman Cancer Institute, University of Utah School of Medicine \\ ${ }^{2}$ Department of Dermatology, University of Utah Health Sciences Center, Salt Lake City \\ * These authors contributed equally
}

Correspondence to: Rodney A. Stewart at rodney.stewart@hci.utah.edu

URL: https://www.jove.com/video/55712

DOI: doi: $10.3791 / 55712$

Keywords: Cancer Research, Issue 123, Transplantation, zebrafish, chemical, screens, drug, invasion, pediatric, cancer, tumors, brain

Date Published: 5/17/2017

Citation: Casey, M.J., Modzelewska, K., Anderson, D., Goodman, J., Boer, E.F., Jimenez, L., Grossman, D., Stewart, R.A. Transplantation of Zebrafish Pediatric Brain Tumors into Immune-competent Hosts for Long-term Study of Tumor Cell Behavior and Drug Response. J. Vis. Exp. (123), e55712, doi:10.3791/55712 (2017).

\section{Abstract}

Tumor cell transplantation is an important technique to define the mechanisms controlling cancer cell growth, migration, and host response, as well as to assess potential patient response to therapy. Current methods largely depend on using syngeneic or immune-compromised animals to avoid rejection of the tumor graft. Such methods require the use of specific genetic strains that often prevent the analysis of immune-tumor cell interactions and/or are limited to specific genetic backgrounds. An alternative method in zebrafish takes advantage of an incompletely developed immune system in the embryonic brain before 3 days, where tumor cells are transplanted for use in short-term assays (i.e., 3 to 10 days). However, these methods cause host lethality, which prevents the long-term study of tumor cell behavior and drug response. This protocol describes a simple and efficient method for the long-term orthotopic transplantation of zebrafish brain tumor tissue into the fourth ventricle of a 2-day-old immune-competent zebrafish. This method allows: 1) long-term study of tumor cell behaviors, such as invasion and dissemination; 2 ) durable tumor response to drugs; and 3) re-transplantation of tumors for the study of tumor evolution and/or the impact of different host genetic backgrounds. In summary, this technique allows cancer researchers to assess engraftment, invasion, and growth at distant sites, as well as to perform chemical screens and cell competition assays over many months. This protocol can be extended to studies of other tumor types and can be used to elucidate mechanisms of chemoresistance and metastasis.

\section{Video Link}

The video component of this article can be found at https://www.jove.com/video/55712/

\section{Introduction}

The transplantation of tumor cells into immune-compromised animals, particularly mouse xenografts, is a widely-used technique used to study mechanisms controlling cancer cell proliferation ${ }^{1,2}$, survival, invasion, and metastasis ${ }^{3,4}$, as well as to provide a platform for screening drugs $^{5,6,7}$. More recently, the transplantation of primary tumor samples into immune-compromised mice has been used to generate patientderived xenograft (PDX) models for diagnostic and preclinical drug screening purposes and is the backbone of the personalized medicine initiative $^{8,9,10,11}$. However, significant evidence shows that modulating the immune system can have a dramatic impact on tumor behavior and patient outcome ${ }^{12,13}$. This has driven the redesign of xenograft-based techniques to include "humanized" mice, in which the immune system of the mouse is reconstituted by co-transplanting human immune cells with tumor cells. However, this approach is still technically challenging, with variable reproducibility and toxicities associated with the technique, in addition to the significant cost ${ }^{14,15}$. Thus, new transplantation techniques in immune-competent animals are needed to accelerate the discovery of immune- and tumor-specific mechanisms of cancer progression and drug response.

Zebrafish are an alternative animal model for the study of human cancer, with over 20 cancer models now established ${ }^{16}$, including highly malignant brain ${ }^{17}$, melanoma ${ }^{18,19,20}$, and pancreatic cancers ${ }^{21,22}$, as well as many leukemias ${ }^{23,24,25,26,27}$. Two attributes of the zebrafish system make it particularly amenable for cancer research: 1) the optical clarity of translucent animals allows for the direct visualization of cancer cell behaviors (i.e., proliferation, survival, invasion, and dissemination) using simple microscopy techniques and 2) female zebrafish can produce up to 200 embryos per day, allowing for the rapid scaling of animal numbers for genetic or drug screening at a low cost. In addition, the cancer genomes of zebrafish and humans are highly conserved (including oncogenes and tumor-suppressor genes) ${ }^{28}$, allowing mechanistic and drug discoveries to be rapidly translated to mammalian systems. These attributes also make the zebrafish an ideal animal model for transplantation techniques, which take advantage of the imaging, scalability, and low cost of the system.

Previous tumor transplantation studies in immune-compromised zebrafish have facilitated the identification of self-renewal capabilities, tumor malignancy, and invasion/dissemination ${ }^{11,29}$. Short-term studies of tumor cell behavior can be conducted following transplantation into $\gamma^{-}$ 
irradiated adults, whose immune systems are effectively suppressed for $\sim 20$ days ${ }^{11,30}$. The treatment of adult zebrafish with dexamethasone suppresses B- and T-cells for up to 30 days before rejection occurs ${ }^{31}$. Another less common strategy employs clonal zebrafish strains that enable long-term investigations in an immune-competent hos ${ }^{32}$. However, only a limited number of clonal strains have been generated and are difficult to maintain due to low fecundity. In addition, most of the established zebrafish tumor models are generated in other genetic backgrounds, so these tumors cannot be transplanted into the clonal strains without suppressing the immune system ${ }^{11,33,34}$. More recent approaches to improve long-term transplantation studies include the development of the rag $2^{E 450 f s}$ mutant line, with compromised B-and T-cell functions, which has been used to successfully transplant multiple cancers ${ }^{35,36}$. To circumvent the requirement for clonal zebrafish lines or an immunecompromised host, a number of groups have used early-stage embryos (i.e., $>72 \mathrm{~h}$ post-fertilization (hpf)) for human tumor cell transplantation, as these embryos have not yet fully developed an adaptive immune system ${ }^{37,38,39,40,41,42,43}$. However, these methods are limited to the short-term analysis of tumor cell behavior or drug response (usually less than 2 weeks) because the human cancer cells or the transplantation technique itself kills the host, preventing long-term studies and re-transplantation.

This protocol details a modified embryonic transplantation method into the lumen of the fourth ventricle of a 2-day-post-fertilization (dpf) embryo brain. It minimizes toxicity to the host and can be combined with zebrafish brain tumor models for the long-term engraftment of tumor cells. Thus, this technique allows for the re-transplantation of tumor cells into new hosts over many generations, facilitating future studies on tumor heterogeneity, host/immune responses, drug responses, or metastatic potential. This method is also simple, efficient, and scalable, as up to 300 transplantations can be performed by a single user per day, with up to $90 \%$ engraftment. This allows for the rapid propagation of single primary tumors into hundreds of embryos at $2 \mathrm{dpf}$ for genetic or drug screening projects or to directly visualize brain tumor cell behavior in different host backgrounds over many months.

Protocol

All animal procedures were approved and followed the animal care guidelines of the University of Utah Institutional Animal Care and Use Committee (IACUC\#16-03019).

\section{Preparing the Equipment for the Microinjection}

\section{Preparing the injection plate.}

1. Prepare a $50 \mathrm{~mL}$ solution of $1.2 \%$ agarose dissolved in egg water $(0.6 \mathrm{~g} / \mathrm{L}$ aquarium salt in reverse osmosis water $+0.01 \mathrm{mg} / \mathrm{L}$ methylene blue). Boil the solution until the agarose dissolves and then supplement by adding $0.05 \mathrm{mg} / \mathrm{L}$ methylene blue. Pour $25 \mathrm{~mL}$ of the final solution into a $10 \mathrm{~cm}$ Petri dish and allow it to solidify. Place the remaining $25 \mathrm{~mL}$ of agarose solution in a $42{ }^{\circ} \mathrm{C}$ water bath until ready for step 1.1.2.

2. Set a 2 inch diameter beaker in the center of the solidified surface and pour the remaining $25 \mathrm{~mL}$ of $1.2 \%$ agarose.

NOTE: This creates an injection surface on the periphery of the plate and a core in the center, which provides an area to test needle size and tumor suspension consistency.

1. Maintain the injection plate at $28^{\circ} \mathrm{C}$ for at least $30 \mathrm{~min}$ prior to injection or at $4{ }^{\circ} \mathrm{C}$ for long-term storage.

2. Preparing injection needles.

1. Make the needles by pulling $10 \mathrm{~cm}$ capillaries with an outer dimension of $1.2 \mathrm{~mm}$ and an inner dimension of $0.9 \mathrm{~mm}$ (without a filament) into two needles on a needle puller.

NOTE: Each needle should measure $6 \mathrm{~cm}$ in total length, where approximately $1.5 \mathrm{~cm}$ of the end is tapered.

2. Carefully place the needle on a microscope slide wrapped in plastic paraffin film. Use a razor blade to cut the end of the needle at a $45^{\circ}$ angle to create a needle with an opening at the tip.

NOTE: Typically, $0.1 \mathrm{~mm}$ to $0.3 \mathrm{~mm}$ of the end of the needle is removed. A beveled tip is not required to perform the injections.

3. Prepare the microinjection setup.

1. Arrange a standard microinjection setup, including a stereomicroscope, a manipulator, and a microinjector for the transplantation.

\section{Preparing Embryos for Transplantation}

1. Collect up to 500 mitfa $^{\text {w2 }}$ embryos ${ }^{44}$ (or embryos of any other zebrafish genotypes as defined by the user) as described ${ }^{45}$ and maintain in a $28^{\circ} \mathrm{C}$ incubator.

2. Dechorionate the embryos at $24 \mathrm{hpf}$ by adding $100 \mu \mathrm{L}$ of $10 \mathrm{mg} / \mathrm{mL}$ protease cocktail solution diluted in egg water and gently rock for approximately $20 \mathrm{~min}$. Rinse the embryos 3 times with fresh egg water to remove the residual protease.

3. Screen the embryos at $48 \mathrm{hpf}$ for the appropriate developmental stage, as defined by standard staging series criteria ${ }^{45}$. Note: Only correctly staged and morphologically normal embryos should be used for the transplantation.

4. Anesthetize approximately twenty 48 -hpf embryos in egg water containing $0.002 \%$ Tricaine-S in a petri dish. Confirm proper anesthesia by observing the cessation of movement.

\section{Making a Tumor Cell Suspension}

NOTE: Zebrafish brain tumor models can be generated by genetically engineering combinations of oncogenes and tumor suppressor genes $^{17,46,47}$. Here, the sox10 promoter-driven NRAS $S^{W T}, p 53^{\text {zdf1/zdf1 }}$ CNS-PNET zebrafish model was used, as recently described ${ }^{17}$.

1. Euthanize a brain tumor-bearing fish whose tumor burden is approximately $20 \%$ of the total body size using a $0.4 \%$ Tricaine-S overdose followed by immersion in ice water according to IACUC-approved protocols. 
2. Dissect the fluorescently labeled tumor with a razor blade and tweezers under a fluorescence microscope using sterile technique and place it in $5 \mathrm{~mL}$ of $1 \times$ phosphate-buffered saline (PBS).

NOTE: Exact dissection methods will be user-defined and dependent on tumor type and tissue location. See the listed references for detailed procedures for the dissection of different zebrafish organs and brain tumors ${ }^{17,48}$.

3. Manually disrupt the tumor mass using a p1000 pipette until a uniform, cloudy solution develops. Remove large particulates with a pipette tip or using a $40 \mu \mathrm{m}$ cell strainer (this is tumor-dependent). Centrifuge the suspension at room temperature for $5 \mathrm{~min}$ at $290 \mathrm{xg}$ and remove the supernatant.

Note: Fluorescence-activated cell sorting (FACS) is not performed to isolate tumor cells. This allows for the transplantation of a heterogeneous population of stromal, immune, and tumor cells.

4. Resuspend the tumor cell pellet in $100 \mu \mathrm{L}$ of sterile PBS and transfer it to a $1.5 \mathrm{~mL}$ microcentrifuge tube. Take $5 \mu \mathrm{L}$ of the cell suspension and dilute it in $250 \mu \mathrm{L}$ of PBS. Count the number of cells using a hemocytometer. Centrifuge the suspension for $3 \mathrm{~min}$ at $290 \mathrm{xg}$, remove the supernatant, and resuspend the pellet in sterile PBS to obtain the desired cell concentration.

NOTE: Typically, cells resuspended in $30-40 \mu \mathrm{L}$ of sterile PBS yield a solution that is viscous and opaque (cell concentration $\geq 100 \mathrm{cells} / \mathrm{nL}$ ) and tend to generate the most successful transplants. Cell viability is not assessed.

5. Store the tumor suspension on a $28^{\circ} \mathrm{C}$ heat block during the transplantation procedure.

\section{Injecting the Tumor Suspension into the Fourth Ventricle of a 2-dpf Embryo}

1. Transfer 10-20 anesthetized embryos (from step 2.4) to the periphery of the injection plate using a transfer pipette; the embryos should fall laterally onto the injection plate, with the ventricles clearly visible and accessible. Use an angled probe to adjust the embryos as needed and position them away from the outer edge of the injection plate.

2. Load $1-2 \mu \mathrm{L}$ of the tumor cell suspension into the injection needle using gel loading pipette tips and insert the needle into the manipulator.

3. Manually lower the manipulator, holding the needle at a $45^{\circ}$ angle. Adjust the knobs on the micromanipulator in the $x, y$, and $z$ directions until the needle is just above and approximately $5 \mathrm{~mm}$ to the right of the embryo head. Look through the eyepieces of the stereomicroscope and slowly adjust the micromanipulator in the $x$-direction until the needle pierces the fourth ventricle of the embryo. Do not allow the needle to pierce the heart or the yolk.

1. Push the foot pedal attached to the microinjector to inject the tumor cell suspension.

NOTE: The injection pressure and time will vary based on needle bore size, but a good starting point is $5-10$ PSI and $50-100 \mathrm{~ms}$. The volume of injected tumor cells can be assessed with standard oil drop techniques through injection into mineral oil, if desired ${ }^{49}$. Injections of $500 \mathrm{pL}$ with a drop diameter of $0.1 \mathrm{~mm}$ typically give the best results.

4. Gently rinse the injected embryos off the injection plate and into a petri dish using fresh egg water. Maintain the embryos in egg water for the remaining procedures or until they are grown in tanks (as described in step 4.9.1).

5. Inspect the injected embryos under a fluorescence stereomicroscope. Confirm that the injection pressure, angle, needle size, and cell suspension viscosity result in tumor cells filling $25-50 \%$ of the ventricle space. Adjust these parameters as necessary.

6. Repeat steps 4.1-4.5 for any remaining embryos. Anesthetize the additional embryos as needed (described in step 2.4). NOTE: An experienced user can inject up to 300 embryos over 3-4 h.

7. Place the embryos back in the $28^{\circ} \mathrm{C}$ incubator overnight.

8. The next day, assess embryo survival by examining morphological and physiological features, such as normal heart and brain development, as described ${ }^{45}$.

9. For screening, anesthetize the embryos, as described in step 2.4 , at 1 day post-transplant (dpt).

1. Use a transfer pipette to add $4 \%$ methylcellulose onto the middle of a petri dish and add the embryos to the methylcellulose drop. Orient the embryos (ventral side facing the light source) using an angled probe and screen for a consistent engraftment size using a fluorescence microscope. Remove any embryos with tumor cells not confined to the ventricle or those that contain tumor cells emcompassing less than $25 \%$ of the ventricle space.

Note: Consistent engraftment is defined as embryos with a single tumor mass that encompasses $25-50 \%$ of the ventricle space ${ }^{17}$. For screening purposes, use a fluorescence stereomicroscope with a $1 \mathrm{X}$ objective, a 0.15 numerical aperture, a magnification ranging from $0.7-1.6 \mathrm{X}$, and an exposure time of $50 \mathrm{~ms}$ to $1 \mathrm{~s}$. For all images, utilize the bright-field channel in addition to either the GFP or RFP channel.

2. For maintenance of tumors, place the transplanted embryos in tanks to grow ${ }^{45}$ at $8 \mathrm{dpf}(6 \mathrm{dpt})$.

\section{Imaging Transplanted Embryos for Cellular Behaviors}

1. Anesthetize the embryos at 3-8 dpf (1-6 dpt) and mount (dorsal side facing the coverslip) in $1.2 \%$ low-melt agarose. Perform time-lapse imaging of the mounted embryos using a laser-scanning confocal microscope for the desired length of time to visualize cellular behaviors, such as migration, cell division, or cell-cell interactions, as needed ${ }^{38,43,53}$.

NOTE: Use an aspect ratio of $1,024 \times 1,024$, a scan speed of $8 \mu \mathrm{s} /$ pixel. Employ scan averaging and define the $z$-depth dimensions to ensure the capture of the entire tumor mass $(1,000-1,500 \mu \mathrm{m})$. Additionally, perform 4-h time-lapse experiments, acquiring an image every 10-15 min using a $40 \mathrm{x}$ objective, to monitor invasive behaviors. The detector sensitivity will vary depending upon the intensity of the tumor mass, and the laser power will be microscope-dependent, but it is generally recommended to be between 15 and $40 \%$.

2. Once the imaging is complete, free the mounted embryo from the agarose using an angled probe and transfer it to a petri dish with fresh egg water.

\section{Drug Administration to Transplanted Embryos and the Assessment of Tumor Burden ${ }^{17}$}

1. Anesthetize embryos at $3 \mathrm{dpf}$ and orient them in $4 \%$ methylcellulose, with the ventral side facing the light source. Image the pre-treatment embryos using a fluorescence microscope ${ }^{17}$. 
2. Place the transplanted embryos at $3 \mathrm{dpf}$ in a 12-well plate, with 10 embryos per well in $0.5 \mathrm{~mL}$ of egg water. Add the appropriate amount of drug to obtain the desired final concentration (e.g., $50 \mu \mathrm{M}$ MEK inhibitor) and return the embryos to a $28^{\circ} \mathrm{C}$ incubator. The next day, remove the drug-treated egg water with a thin-bore transfer pipette and add fresh treatment. Repeat this daily until the embryos reach $8 \mathrm{dpf}$.

3. At $8 \mathrm{dpf}$, remove the embryos from drug treatment and place them in fresh egg water. Anesthetize the embryos and orient (ventral side facing the light source) in $4 \%$ methylcellulose. Image embryos post-treatment using a fluorescence microscope. Quantify the tumor area using appropriate software, as described ${ }^{17}$.

4. Separate the embryos as appropriate per treatment and place them in tanks to grow. After 4-9 weeks, anesthetize the fish and assess them for overall tumor burden using a standard fluorescence stereomicroscope, as described ${ }^{17}$.

\section{Representative Results}

\section{Tumor transplantation and engraftment}

A schematized outline of the procedure is represented in Figure 1. Fluorescently labeled tumor cells are extracted from the primary organ site, and a single-cell suspension is generated for transplantation into the fourth ventricle of 2-dpf mitfa ${ }^{\text {w2 }}$ zebrafish embryos ${ }^{44}$. Figure 2 shows the representative results for this method using a zebrafish model of central nervous system primitive neural ectodermal tumor (CNS-PNET), in which the sox10 promoter drives expression of wildtype NRAS or activated NRAS fused to mCherry in p53-deficient oligoneural precursor cells $^{17}$. The mitfa ${ }^{\text {w2 }}$ zebrafish ${ }^{44}$ mutant, which lacks melanophores, were used to more readily visualize the tumor cells after transplantation and/or into adulthood. Other pigment mutants could also be used to provide additional imaging clarity, such as Casper ${ }^{50}$. As early as $24 \mathrm{~h}$ posttransplantation, tumor cells can be seen invading into surrounding brain tissue (Figure 2A, $3 \mathrm{dpf}$, arrows). Tumor grafts continue to grow in the ventricle and surrounding brain tissue over the next week (Figure 2A, $8 \mathrm{dpf}$ ). At this time, fluorescence can also be observed in the region of the kidneys (Figure 2A, asterisks). This may be due to the kidneys filtering the cellular debris from the transplantation, which is consistent with prior studies that use fluorescent dyes as an assay to evaluate kidney function ${ }^{51}$. Tumor cells continue to proliferate and invade, so by $28 \mathrm{dpf}$, a tumor mass can be seen throughout the zebrafish brain (Figure 2A, $28 \mathrm{dpf}$ ).

This technique allows for the rapid transplantation of tumor cells into hundreds of embryos with reproducible consistency, as the injection site can be targeted accurately with minimal damage to the host. A representative group of embryos transplanted with mCherry-labeled brain tumor cells is shown in the top panel of Figure 2B. Tumor engraftment is typically achieved in $80-90 \%$ of embryos, and tumor transplants persist into adulthood; three representative examples are shown in the bottom panels of Figure 2B. This allows for the tumor transplants to be harvested and frozen or re-transplanted over several generations.

\section{Tumor invasion and drug responses}

Figure 3A shows a co-transplantation scheme in which two different tumors labeled with either GFP (Tumor A) or mCherry (Tumor B) are harvested by whole-tumor dissociation (note that FACS is not used here, but FACS-sorted tumor cells could be used if needed). Tumor A and B can be from different locations, induced with different oncogenes, or induced in different genetic backgrounds. Each tumor is processed into a single-cell suspension, and tumor cells are then mixed together at a 1:1 ratio and transplanted into the 2-dpf embryo. Tumor properties, such as engraftment, growth, invasion, and dissemination of Tumor A versus Tumor B can then be observed in the same host that allows internal controls for the technique and genetic background.

In Figure 3B, an NRAS-driven, mCherry-labeled zebrafish CNS-PNET from the optic tectum and an GFP-labeled CNS-PNET from the cerebellum were harvested and processed into single-cell suspensions. Cells were counted, and an equal number of cells from each tumor were mixed together and transplanted into 2-dpf embryos. Four days after transplantation, the embryos were imaged with confocal microscopy. Shown is a representative embryo demonstrating that the tectum tumor cells (red) migrate more extensively into the host brain (arrows) than the cerebellar tumor.

Embryos transplanted with fluorescently labeled tumor cells can be used in drug treatment regimens to identify compounds that inhibit tumor growth. Figure $\mathbf{3 C}$ is a typical timeline for treating and imaging tumor transplants. $24 \mathrm{~h}$ post-transplantation, embryos are accessed for consistent engraftment size and split into treatment groups. In the provided example, embryos transplanted with NRAS-driven, mCherry-labeled zebrafish CNS-PNETs are treated with $50 \mu \mathrm{M}$ dimethyl sulfoxide (DMSO) or MEK inhibitor (AZD6244). Embryos are treated for 5 days and imaged at $8 \mathrm{dpf}$, as outlined in Figure 3C. Figure 3D (left panels) shows representative animals from the two treatment groups. MEK inhibitor-treated embryos have a significantly lower amount of fluorescence than those treated with DMSO, as described ${ }^{17}$. After the animals are imaged, they are transferred to tanks without drugs and monitored for tumor growth for two months. In the provided example, the MEK inhibitor treatment results in a durable response in the adult fish (Figure 3D, right panels). For further details on the drug treatment, imaging, and quantification, see reference ${ }^{17}$. 


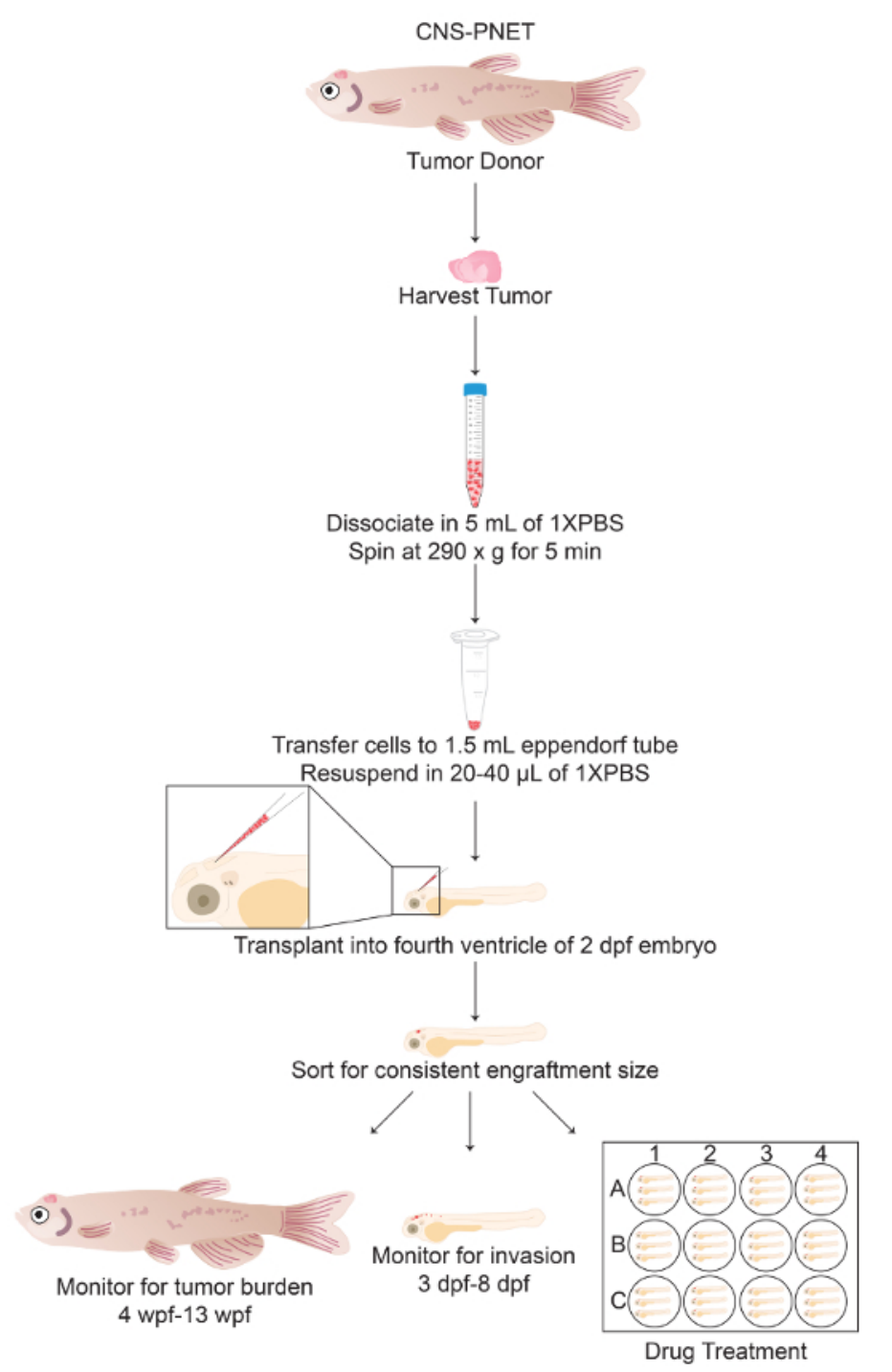

Figure 1: Protocol Schematic of Tumor Cell Transplantation into the Fourth Ventricle of 2-dpf Zebrafish Embryos. General schematic of sections 3 through 4 of the protocol including alternative endpoint analyses (sections 4.9-6). Please click here to view a larger version of this figure. 
A.
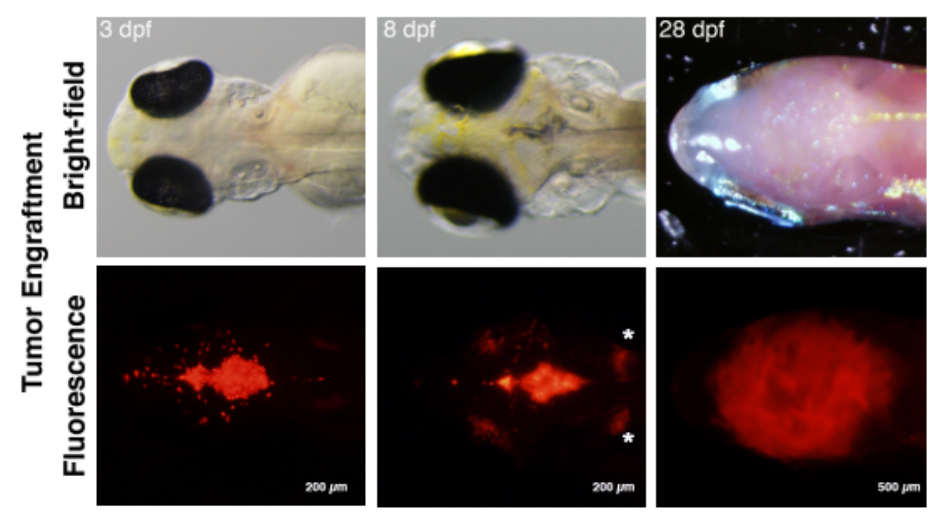

B.
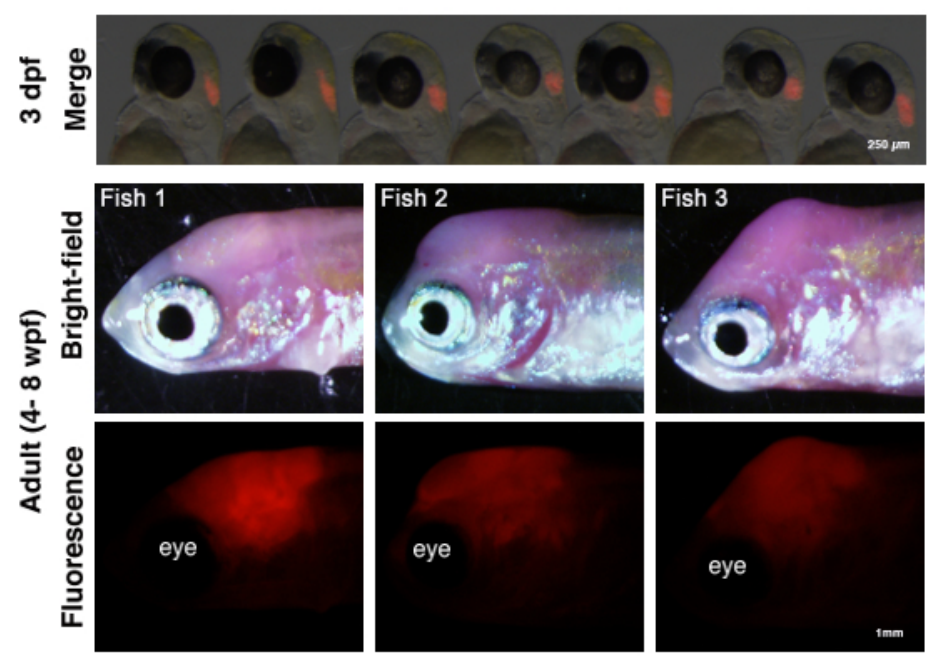

Figure 2: Tumor Engraftment and Progression. (A) Zebrafish primary CNS-PNET ${ }^{17}$ tumor cells labeled with mCherry were transplanted into the fourth ventricle of 2-dpf embryos. At $3 \mathrm{dpf}$, tumor cells are seen invading into surrounding brain tissue (arrows). At $8 \mathrm{dpf}$, tumor cells are growing in the ventricle; red tumor cells are also present in the surrounding brain tissue and kidneys (asterisks). At $28 \mathrm{dpf}$, tumors cells have invaded and grow throughout the host brain. (B) Top panel. Seven 3-dpf embryos transplanted with mCherry-labeled zebrafish brain tumor cells. Tumor cells can be transplanted consistently into hundreds of embryos, with high engraftment rates (i.e., typically $85 / 100$ embryos). Middle and bottom panels. Three representative adult fish with transplanted tumors 4-8 weeks post-fertilization (wpf). Tumors can be visualized by fluorescence microscopy. Please click here to view a larger version of this figure. 
A.

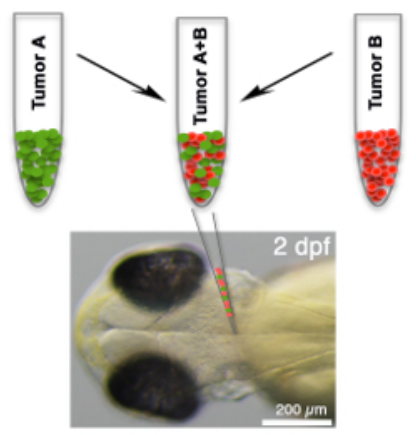

B.

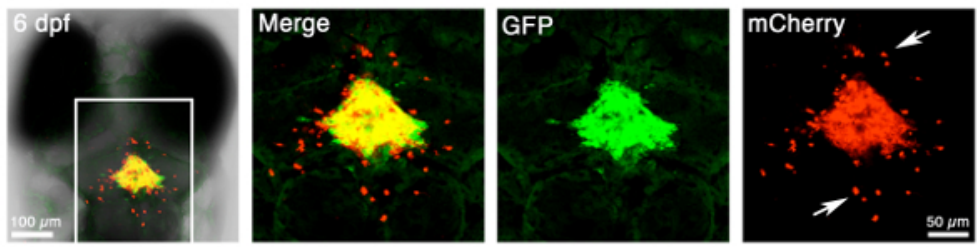

C.

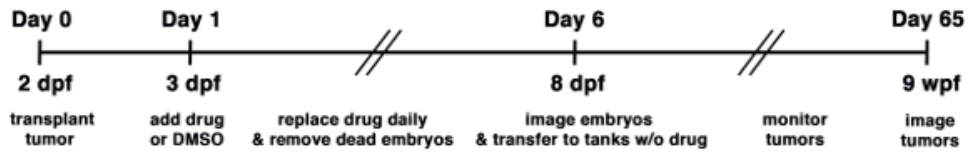

D.

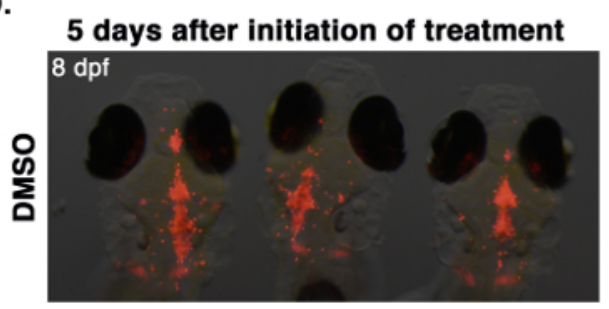

8 weeks post treatment
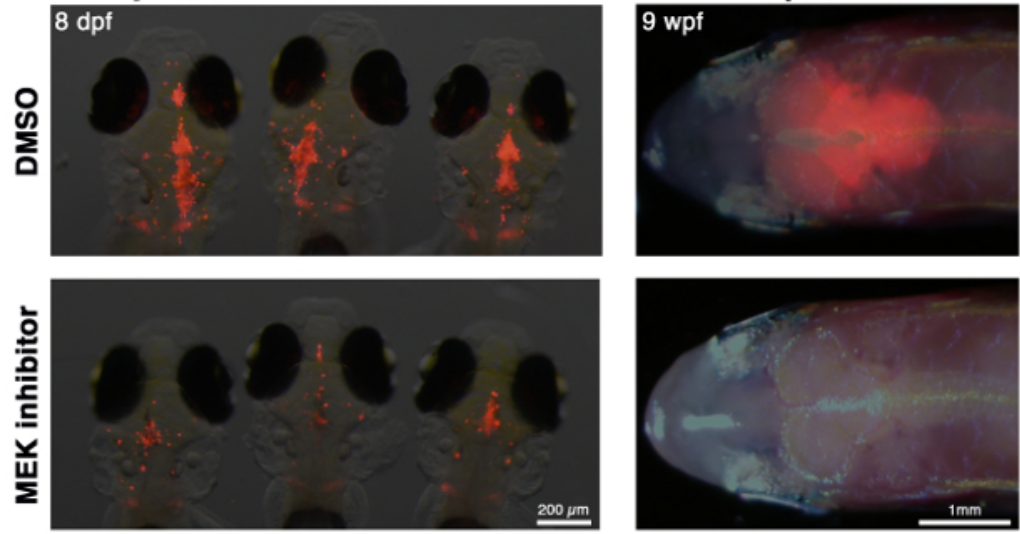

Figure 3: Comparing Tumor Invasion and Drug Response. (A) CNS-PNETs ${ }^{17}$ from the optic tectum or cerebellum are labeled with mCherry or GFP, respectively, processed into a single-cell suspension, mixed together at a 1:1 ratio, and co-transplanted into 2-dpf embryos. (B) A 6-dpf embryo co-transplanted with a mixed suspension of GFP-labeled cerebellar tumor cells and mCherry-labeled tumor cells from the optic tectum. Differences in the invasive properties of each tumor in the same recipient can be observed using fluorescence microscopy. In this example, mCherry-labeled tumor cells migrate more extensively than those labeled with GFP (arrows). (C) Timeline for drug treatment on embryos transplanted with tumor cells. (D) Images of representative animals at the end of the treatment regimen ( $8 \mathrm{dpf})$ and at 8 weeks post-treatment (8 wpf), with either a DMSO control or a $50 \mu \mathrm{M}$ MEK inhibitor. Tumor burden can be measured by quantifying fluorescence, as described ${ }^{17}$. In this experiment, the growth of mCherry-labeled tumor cells is reduced in MEK inhibitor-treated embryos and translates into a durable response in the adult. Please click here to view a larger version of this figure.

\section{Discussion}

This protocol details a simple and efficient transplantation assay that involves the injection of zebrafish tumor cells into the ventricle of a 2dpf embryo that will develop a fully competent immune system. So far, zebrafish CNS-PNET ${ }^{17}$ and melanoma (data not shown) have been successfully transplanted for long-term studies of tumor cell behavior and invasion. The critical steps of this protocol include ensuring the appropriate needle bore size and the proper tumor cell suspension and adequately anesthetizing host embryos. For each individual researcher, further optimization of this technique may include the adjustment of tumor cell concentration, injection pressure, and embryo orientation. In addition, there can be heterogeneity in the viscosity of suspensions originating from different tumors, so different tumor types may be more or less difficult to re-suspend and transplant. However, by adjusting the needle bore size and by using different dilutions of the tumor suspension, it is possible to overcome difficulties associated with tumor viscosities. 
In our experience, the most common reasons for sparse cells/inconsistent cell masses in the ventricle are as follows: 1) the tumor cell suspension is too dilute; 2) the needle bore size is too small; 3 ) the injection time and pressure are too low; and/or 4) remaining tissue were not filtered from the tumor and are lodged in the needle. When the tumor cell suspension flows out of the ventricle after injection, it is likely due to: 1 ) the placement of the needle against the floor of the ventricle; 2) a high injection pressure and time; and/or 3) a needle bore size that is too large. Low survival of transplanted embryos may be caused by: 1) anesthetizing embryos for an extended period of time; 2) leaving embryos on the injection plate to dry; 3 ) the injection of air bubbles into the ventricle; and/or 4) piercing vital organs, such as the brain and heart, because the needle went through the ventricle.

Previous methods of tumor transplantation in the adult or embryonic zebrafish brain rely upon immunosuppression in adults (genetically, pharmacologically, or via radiation) or are limited to short-term studies of human or mouse cells in embryos ${ }^{38,43,52,53,54}$. For example, mouse brain tumors can be injected intranasally into dexamethasone-immunosuppressed, 30-dpf, juvenile zebrafish for use in short term ( 2 days) pre-clinical drug assays ${ }^{54}$. However, the site of injection in these experiments is obscured by the skull and brain tissue and likely results in damage to normal brain tissue, which reduces host viability and engraftment efficiency. Another recently described method involves the injection of human glioblastoma cell lines into the midbrain region of zebrafish embryos, which enabled the short-term analysis of growth, invasion, and drug response ${ }^{43}$. Again, the precise location of the injection site is variable and likely damages normal tissue. Thus, previous methods of embryonic brain transplantations often compromise the viability of the hosts, limiting these studies to short-term analysis (i.e., 2 to 14 days), causing increased variability between individual injections due to obscured injection sites, and preventing the long-term analysis of cell behaviors and drug responses or of re-transplantation across multiple generations.

The method described here addresses current limitations in zebrafish embryonic and immune-compromised transplantation techniques by allowing researchers to: 1) reproducibly inject into the same location, with minimal damage to surrounding tissue; 2) directly visualize the site of injection to maximize engraftment efficiency; 3 ) transplant hundreds of embryos per day; 4) allow tumors to grow in immune-competent animals; 5) allow for the monitoring of tumor cell behavior and durable drug responses over the life of the zebrafish; and 6) allow for the re-transplantation of tumors over many generations for potential studies on tumor evolution or drug relapse mechanisms. Furthermore, this protocol enables researchers to utilize any zebrafish genotype for the assessment of host response, including different immune populations. These attributes make this method easily adaptable by any lab that already performs standard microinjections in zebrafish. Finally, while this method is ideal for orthotopic injections of zebrafish brain tumors, when transplanting other tumor types, such as liver or pancreatic, the orthotopic site may be more important than immune competence (e.g., if a researcher is studying the effects of the stromal microenvironment on tumor growth). In this scenario, the newly developed, immune-deficient zebrafish models may be more appropriate for performing orthotopic tumor transplantations ${ }^{11}$.

This protocol has been used to perform tumor cell competition assays and to inject dual-labeled tumors. A potential treatment strategy for the assessment of the effectiveness of chemical compounds on tumorigenesis, which involves the treatment of embryos post-transplant via the addition of the drug to water, was also discussed. A method for the ex vivo treatment of tumor cells prior to transplantation was also previously reported $^{17}$. Additionally, transplanted tumors have been pooled for multiple rounds of re-transplantation, which will be beneficial to studies of tumor evolution and chemoresistance ${ }^{17}$. Currently, pre-clinical studies are dependent on mouse xenografts to assess the efficacy of potential compounds. However, these studies are time-consuming and costly. Considering the high degree of conservation of oncogenic signaling pathways between zebrafish and humans ${ }^{28}$, it is to be expected that this method will complement conventional mouse and human cell studies to allow for the more rapid identification of effective compounds entering pre-clinical and clinical trials. Ultimately, this method could prove to be useful for the rapid chemical screening of primary patient tumors, which could further propel the personalized medicine initiative. However, conditions for the long-term growth of human cells in zebrafish (adult or embryo) still need to be identified.

Disclosures

The authors have nothing to disclose.

\section{Acknowledgements}

We thank the two reviewers for excellent suggestions and improvements to the manuscript. We also thank Huntsman Cancer Institute/University of Utah for the animal husbandry and maintenance. This work was funded by the American Cancer Society (\#124250- RSG-13-025-01-CSM), an $\mathrm{NIH}$ grant (P30 CA042014 CRR program), the University of Utah Seed Grant, and the Huntsman Cancer Foundation.

\section{References}

1. Shaw, T. J., Senterman, M. K., Dawson, K., Crane, C. A., \& Vanderhyden, B. C. Characterization of intraperitoneal, orthotopic, and metastatic xenograft models of human ovarian cancer. Mol Ther. 10 (6), 1032-1042 (2004).

2. Stephen, R. M. et al. Monitoring the development of xenograft triple-negative breast cancer models using diffusion-weighted magnetic resonance imaging. Exp Biol Med (Maywood). 237 (11), 1273-1280 (2012).

3. Deroose, C. M. et al. Multimodality imaging of tumor xenografts and metastases in mice with combined small-animal PET, small-animal CT, and bioluminescence imaging. J Nucl Med. 48 (2), 295-303 (2007).

4. Saxena, M., \& Christofori, G. Rebuilding cancer metastasis in the mouse. Mol Oncol. 7 (2), 283-296 (2013).

5. Johnson, J. I. et al. Relationships between drug activity in $\mathrm{NCl}$ preclinical in vitro and in vivo models and early clinical trials. $\mathrm{Br} \mathrm{J}$ Cancer. 84 (10), 1424-1431 (2001).

6. Kerbel, R. S. Human tumor xenografts as predictive preclinical models for anticancer drug activity in humans - Better than commonly perceived - But they can be improved. Cancer Biology \& Therapy. 2 (4), S134-S139 (2003).

7. Scholz, C. C., Berger, D. P., Winterhalter, B. R., Henss, H., \& Fiebig, H. H. Correlation of Drug Response in Patients and in the ClonogenicAssay with Solid Human Tumor Xenografts. Eur J Cancer. 26 (8), 901-905 (1990). 
8. Siolas, D., \& Hannon, G. J. Patient-derived tumor xenografts: transforming clinical samples into mouse models. Cancer Res. 73 (17), 5315-5319 (2013).

9. Cho, S. Y. et al. An Integrative Approach to Precision Cancer Medicine Using Patient-Derived Xenografts. Mol Cells. 39 (2), 77-86 (2016).

10. Hiroshima, Y. et al. Patient-derived mouse models of cancer need to be orthotopic in order to evaluate targeted anti-metastatic therapy. Oncotarget. 7 (44), 71696-71702 (2016).

11. Moore, J. C., \& Langenau, D. M. Allograft Cancer Cell Transplantation in Zebrafish. Adv Exp Med Biol. 916 265-287 (2016).

12. Hanahan, D., \& Weinberg, R. A. Hallmarks of cancer: the next generation. Cell. 144 (5), 646-674 (2011).

13. Dranoff, G. Experimental mouse tumour models: what can be learnt about human cancer immunology? Nat Rev Immunol. 12 (1), $61-66$ (2011).

14. Richmond, A., \& Su, Y. Mouse xenograft models vs GEM models for human cancer therapeutics. Dis Model Mech. 1 (2-3), $78-82$ (2008).

15. Bernard, D., Peakman, M., \& Hayday, A. C. Establishing humanized mice using stem cells: maximizing the potential. Clin Exp Immunol. 152 (3), 406-414 (2008).

16. White, R., Rose, K., \& Zon, L. Zebrafish cancer: the state of the art and the path forward. Nat Rev Cancer. 13 (9), $624-636$ (2013).

17. Modzelewska, K. et al. MEK Inhibitors Reverse Growth of Embryonal Brain Tumors Derived from Oligoneural Precursor Cells. Cell Rep. 17 (5), 1255-1264 (2016).

18. Patton, E. E. et al. BRAF mutations are sufficient to promote nevi formation and cooperate with p53 in the genesis of melanoma. Curr Biol. 15 (3), 249-254 (2005).

19. Dovey, M., White, R. M., \& Zon, L. I. Oncogenic NRAS cooperates with p53 loss to generate melanoma in zebrafish. Zebrafish. 6 (4), $397-404$ (2009).

20. Santoriello, C. et al. Kita driven expression of oncogenic HRAS leads to early onset and highly penetrant melanoma in zebrafish. PLoS One. 5 (12), e15170 (2010).

21. Park, S. W. et al. Oncogenic KRAS induces progenitor cell expansion and malignant transformation in zebrafish exocrine pancreas. Gastroenterology. 134 (7), 2080-2090 (2008).

22. Liu, S., \& Leach, S. D. Screening pancreatic oncogenes in zebrafish using the Gal4/UAS system. Methods Cell Biol. 105 367-381 (2011).

23. Langenau, D. M. et al. Myc-induced T cell leukemia in transgenic zebrafish. Science. 299 (5608), 887-890 (2003).

24. Langenau, D. M. et al. Cre/lox-regulated transgenic zebrafish model with conditional myc-induced T cell acute lymphoblastic leukemia. Proc Natl Acad Sci U S A. 102 (17), 6068-6073 (2005).

25. Sabaawy, H. E. et al. TEL-AML1 transgenic zebrafish model of precursor B cell acute lymphoblastic leukemia. Proc Natl Acad Sci U S A. 103 (41), 15166-15171 (2006).

26. Chen, J. et al. NOTCH1-induced T-cell leukemia in transgenic zebrafish. Leukemia. 21 (3), 462-471 (2007).

27. Feng, $\mathrm{H}$. et al. Heat-shock induction of T-cell lymphoma/leukaemia in conditional Cre/lox-regulated transgenic zebrafish. $\mathrm{Br} \mathrm{J} H a e m a t o l .138$ (2), 169-175 (2007).

28. Howe, K. et al. The zebrafish reference genome sequence and its relationship to the human genome. Nature. 496 (7446), $498-503$ (2013)

29. Li, P., White, R. M., \& Zon, L. I. Transplantation in zebrafish. Methods Cell Biol. 105 403-417 (2011).

30. Traver, D. et al. Effects of lethal irradiation in zebrafish and rescue by hematopoietic cell transplantation. Blood. 104 (5), 1298-1305 (2004).

31. Stoletov, K., Montel, V., Lester, R. D., Gonias, S. L., \& Klemke, R. High-resolution imaging of the dynamic tumor cell vascular interface in transparent zebrafish. Proc Natl Acad Sci U S A. 104 (44), 17406-17411 (2007).

32. Streisinger, G., Walker, C., Dower, N., Knauber, D., \& Singer, F. Production of clones of homozygous diploid zebra fish (Brachydanio rerio) Nature. 291 (5813), 293-296 (1981).

33. Mizgirev, I., \& Revskoy, S. Generation of clonal zebrafish lines and transplantable hepatic tumors. Nat Protoc. 5 (3), $383-394$ (2010).

34. Mizgireuv, I. V., \& Revskoy, S. Y. Transplantable tumor lines generated in clonal zebrafish. Cancer Res. 66 (6), $3120-3125$ (2006).

35. Tenente, I. M., Tang, Q., Moore, J. C., \& Langenau, D. M. Normal and malignant muscle cell transplantation into immune compromised adult zebrafish. J Vis Exp. 94 (94) (2014).

36. Tang, Q. et al. Optimized cell transplantation using adult rag2 mutant zebrafish. Nat Methods. 11 (8), 821-824 (2014).

37. Geiger, G. A., Fu, W., \& Kao, G. D. Temozolomide-mediated radiosensitization of human glioma cells in a zebrafish embryonic system. Cancer Res. 68 (9), 3396-3404 (2008).

38. Kitambi, S. S. et al. Vulnerability of glioblastoma cells to catastrophic vacuolization and death induced by a small molecule. Cell. 157 (2), 313-328 (2014).

39. Lally, B. E. et al. Identification and biological evaluation of a novel and potent small molecule radiation sensitizer via an unbiased screen of a chemical library. Cancer Res. 67 (18), 8791-8799 (2007).

40. Vittori, M., Motaln, H., \& Turnsek, T. L. The study of glioma by xenotransplantation in zebrafish early life stages. J Histochem Cytochem. 63 (10), 749-761 (2015).

41. Yang, X. J. et al. A novel zebrafish xenotransplantation model for study of glioma stem cell invasion. PLoS One. 8 (4), e61801 (2013).

42. Zhao, H., Tang, C., Cui, K., Ang, B. T., \& Wong, S. T. A screening platform for glioma growth and invasion using bioluminescence imaging. Laboratory investigation. J Neurosurg. 111 (2), 238-246 (2009).

43. Welker, A. M. et al. Correction: Standardized orthotopic xenografts in zebrafish reveal glioma cell-line-specific characteristics and tumor cell heterogeneity. Dis Model Mech. 9 (9), 1063-1065 (2016).

44. Lister, J. A., Robertson, C. P., Lepage, T., Johnson, S. L., \& Raible, D. W. nacre encodes a zebrafish microphthalmia-related protein that regulates neural-crest-derived pigment cell fate. Development. 126 (17), 3757-3767 (1999).

45. Westerfield, M. The Zebrafish Book. A Guild for the Laboratory Use of Zebrafish (Danio rerio). University of Oregon Press. (2000).

46. Solin, S. L., Shive, H. R., Woolard, K. D., Essner, J. J., \& McGrail, M. Rapid tumor induction in zebrafish by TALEN-mediated somatic inactivation of the retinoblastoma1 tumor suppressor rb1. Sci Rep. 513745 (2015).

47. Ju, B. et al. Oncogenic KRAS promotes malignant brain tumors in zebrafish. Mol Cancer. 1418 (2015).

48. Gupta, T., \& Mullins, M. C. Dissection of organs from the adult zebrafish. J Vis Exp. 37 (37) (2010).

49. Rosen, J. N., Sweeney, M. F., \& Mably, J. D. Microinjection of zebrafish embryos to analyze gene function. J Vis Exp. (25) (2009).

50. White, R. M. et al. Transparent adult zebrafish as a tool for in vivo transplantation analysis. Cell Stem Cell. 2 (2), 183-189 (2008).

51. Christou-Savina, S., Beales, P. L., \& Osborn, D. P. Evaluation of zebrafish kidney function using a fluorescent clearance assay. J Vis Exp. 96 (96), e52540 (2015).

52. Rampazzo, E. et al. Wnt activation promotes neuronal differentiation of glioblastoma. Cell Death Dis. 4 (e500), e500 (2013). 
53. Lal, S., La Du, J., Tanguay, R. L., \& Greenwood, J. A. Calpain 2 is required for the invasion of glioblastoma cells in the zebrafish brain microenvironment. J Neurosci Res. 90 (4), 769-781 (2012).

54. Eden, C. J. et al. Orthotopic models of pediatric brain tumors in zebrafish. Oncogene. 34 (13), 1736-1742 (2015). 